\title{
Experiencia y afrontamiento ante el riesgo aluvional: Un modelo mediacional múltiple
}

\author{
José Sandoval-Díaz; Naxhelli Ruiz-Rivera; David Cuadra-Martínez
}

Cómo citar este artículo:
Sandoval-Díaz, J., Ruiz-Rivera, N., \& Cuadra-Martínez, D. (2021). Experiencia y afrontamiento ante el riesgo aluvional:
Un modelo mediacional múltiple. Acta Colombiana de Psicología, 24(2), 130-143. https://doi.org/10.14718/ACP.2021.24.2.12

Recibido, febrero 12/2021; Concepto de evaluación, mayo 05/2021; Aceptado, mayo 25/2021

\author{
José Sandoval-Díaz ${ }^{1}$ \\ ORCID: https://orcid.org/0000-0001-7247-7113 \\ Universidad del Bío-Bío, Chillán, Chile. \\ Naxhelli Ruiz-Rivera \\ ORCID: https://orcid.org/0000-0003-4948-1557 \\ Universidad Nacional Autónoma de México, Ciudad de México, México. \\ David Cuadra-Martínez \\ ORCID: https://orcid.org/0000-0002-0810-2795 \\ Universidad de Atacama, Copiapó, Chile.
}

\begin{abstract}
Resumen
El incremento de riesgos hidrometereológicos causados por el cambio climático ha llevado consigo la necesidad de fortalecer las capacidades adaptativas de afrontamiento. Bajo este contexto, el objetivo del presente estudio fue esclarecer la relación entre la experiencia directa y las capacidades activas de afrontamiento, considerando tres posibles mediadores: la severidad subjetiva, la percepción del riesgo y la implicancia personal. Para esto, se obtuvo una muestra por conveniencia $(n=490)$ de una ciudad chilena afectada por aluviones. Como resultado, si bien todas las variables correlacionaron directamente con las capacidades activas (entre $r=.13$ y $r=.49$ ), la relación principal solo estuvo mediada por el temor al riesgo y la identificación y posibilidad de acción; por tanto, se concluye que la experiencia directa previa, el miedo y la autoeficacia percibida impulsarían comportamientos precautorios activos frente al riesgo aluvional.

Palabras clave: aluvión, experiencia, percepción del riesgo, capacidades de afrontamiento, modelos de mediación.
\end{abstract}

\section{Experience and coping in the face of alluvial risk: A multiple mediation model}

\begin{abstract}
The increase of hydro-meteorological risks, caused by climate change, has brought with it the need to strengthen adaptive coping capacities. In this context, the aim of the present study was to clarify the relationship between direct experience and active coping capacities, considering three possible mediators: subjective severity, risk perception and personal involvement. For this purpose, a convenience sample $(\mathrm{n}=490)$ was obtained from a Chilean city affected by alluvium. Although all variables directly correlated with active capabilities (between $r=.13$ and $r=.49$ ), the main relationship was only mediated by i) fear for risk and ii) identification and possibility of action. Therefore, it is concluded that previous direct experience, fear and perceived self-efficacy, would drive active precautionary behaviors in the face of alluvial risk.

Keywords: alluvium, experience, risk perception, coping skills, mediation models.
\end{abstract}

\footnotetext{
1 Departamento de Ciencias Sociales, Centro de Estudios Ñuble, Género, Ciudadanía y Territorio(GECiT) de la Universidad del Bío-Bío, Chile. Avenida Andrés Bello 720 (Campus Fernando May), Chillán, Ñuble. jsandoval@ubiobio.cl

Trabajo financiado por la Agencia Nacional de Investigación y Desarrollo (ANID) en el marco de la ejecución del FONDECYT de Iniciación № 11200683 "Riesgos socionaturales intensificados por el cambio climático: Análisis de la vulnerabilidad-resiliencia social a escala local".
} 


\section{Introducción}

El cambio climático se ha convertido en uno de los principales aspectos que profundizan la vulnerabilidad ante desastres socionaturales, razón por la cual se hace necesaria la implementación de estrategias de mitigación y adaptación al riesgo a escala global y local (UN Office for Disaster Risk Reduction [UNDRR], 2015). En este sentido, ante la mayor frecuencia e intensidad de eventos extremos, como lo son el aumento en el nivel del mar, el estrés por calor, el oleaje por tormentas, las sequías, los deslizamientos, la aridez, la contaminación del aire, las precipitaciones extremas y las inundaciones (Intergovernmental Panel on Climate Change [IPCC], 2014), se requiere profundizar el conocimiento respecto a las respuestas humanas ante los eventos críticos, con el fin de comprender la capacidad adaptativa necesaria para la reducción del riesgo de desastre en las ciudades latinoamericanas (Sapiains \& Ugarte, 2017; Siclari, 2020).

Respecto a las amenazas climatológicas, se encuentra que las sequías y las inundaciones representan casi el $90 \%$ de los riesgos socionaturales mundiales, siendo las inundaciones las más frecuentes y destructivas (IPCC, 2014). Solo para el año 2018, el impacto de eventos extremos afectó a 57.3 millones de personas, liderado por las inundaciones, con una afectación de 35.4 millones (UNDRR, 2019).

Para el caso de Chile, de acuerdo con Henríquez et al. (2016), se han registrado 78 eventos de desastres socionaturales entre los años 1984 y 2013, siendo los temporales de lluvia la amenaza más recurrente $(61 \%)$, seguidos por sequías (20\%), nevazones ( $9 \%$ ), heladas ( $7 \%$ ) y finalmente los aluviones - flujo de barro o detrito (fragmentos de roca) con gran poder destructivo, en el cual el agua arrastra el material suelto por laderas, quebradas o cauces-, con solo tres eventos en ese periodo, a los cuales se suman los eventos del norte grande de 2015 y 2017 respectivamente. Este aumento sostenido de aluviones revela la importancia de una comprensión más detallada de cómo los territorios y hogares expuestos afrontan estos eventos extremos (Babcicky \& Seebauer, 2017; Sandoval-Díaz et al., 2020).

Ahora bien, de acuerdo con Grothmann y Reusswig (2006), se han identificado dos factores determinantes para que los hogares implementen medidas de mitigación ante inundaciones: una alta percepción del riesgo (en adelante, PR), y la capacidad autopercibida para afrontarlos.

Para Dzialek (2013), la percepción de riesgos naturales implica juicios, creencias y actitudes intuitivas sobre la probabilidad de ocurrencia, curso y mecanismos implicados ante la amenaza en cuestión, y, de acuerdo con el paradigma psicométrico, la PR presenta dos dimensiones centrales: el temor y el conocimiento (Bodemer \& Gaissmaier, 2015).
Para Slovic (2000), en particular, el temor se vincula al potencial catastrófico, la falta de control percibido y las consecuencias letales, mientras que el conocimiento representa "eventos que se juzgan como inobservables, desconocidos, nuevos y retardados en su manifestación de daño" (p. 226).

Ahora, entre los elementos determinantes de la PR se han considerado distintos factores, y entre ellos destaca, primero, la naturaleza de la amenaza, que se divide en eventos extremos y de manifestación lenta. Para los primeros, como es el caso de los aluviones, se ha concluido que cuanto mayor es la PR, mayor es la probabilidad de tomar acciones preventivas y precautorias (Lindell \& Perry, 2012). No obstante, también se ha encontrado que un mayor grado de PR no implica necesariamente una mayor disposición a tomar medidas de autoseguridad (Siegrist \& Gutscher, 2006).

Un segundo elemento considerado es la implicación personal (en adelante IP), un factor psicosocial definido como la distancia funcional entre el individuo y el riesgo, en términos de predisponer a una acción pertinente y eficaz (Flament \& Rouquette, 2003). Respecto a sus componentes, se ha identificado la valoración de la importancia del problema, la identificación de la relación con el objeto, y el sentimiento de control, en términos de evaluación de capacidad autopercibida (Gruev-Vintila \& Rouquette, 2007). Así, aplicada al campo de riesgo de desastres, la IP condicionaría las prácticas sociales y capacidades de afrontamiento en tanto posibilidad de acción, de manera que una menor IP implicaría escasas acciones o decisiones para mitigar y adaptarse a los riesgos (Zapa-Pérez et al., 2017). Específicamente, para el caso de las inundaciones costeras y repentinas, la IP ha sido considerada como una variable mediadora entre el riesgo percibido y las acciones de protección, prevención y afrontamiento (Navarro et al., 2020; Navarro et al., 2016).

En tercer lugar, estudios relevan las experiencias previas de desastre, que se dividen en experiencia directa de daño, por un lado, y experiencias indirectas o en el rol de espectador, por el otro (Fox et al., 2017); sin embargo, no todas las formas de experiencia están relacionadas con ajustes perceptivos adecuados, pues otros factores también tienen un importante rol de interacción, como lo son la naturaleza de la amenaza y la exposición (Ho et al., 2008). De acuerdo con esto último, varios estudios señalan una fuerte relación entre exposición y riesgo percibido, donde se considera la proximidad como otro de sus elementos clave (Arias et al., 2017; Botzen et al., 2009).

Para el caso de las inundaciones, Kellens et al. (2011) conciben la PR como un análisis de la conciencia, emociones y comportamientos ante la amenaza, que se vincula estrechamente con la proximidad y la exposición. En la misma línea, Muñoz-Duque y Arroyave (2017) encontraron que 
tanto la exposición como la experiencia ante inundaciones son predictores de PR en población colombiana, en particular, en su dimensión afectiva. Asimismo, Raaijmakers et al. (2008) circunscriben la PR a una combinación de tres factores específicos: conciencia, preocupación y preparación. Y, por último, en la revisión sistemática realizada por Lechowska (2018) se identificaron 22 factores frecuentes en las investigaciones empíricas sobre PR ante inundaciones, que se agrupan en: (a) primarios o de influencia directa - preocupación, conciencia, experiencia directa, conocimiento y género-, (b) secundarios o de influencia poco clara - proximidad de amenaza, ubicación del peligro, longitud de residencia, propiedad de la vivienda, edad, educación y preparación-, y, finalmente, (c) intervinientes o vinculadas al contexto.

Sumado a lo disposicional, para afrontar adecuadamente estos eventos extremos resulta necesaria la adquisición y desarrollo de capacidades para anticipar, resistir, recuperarse y adaptarse (Burkett, 2013; UNDRR, 2015); capacidades que incluyen un amplio abanico de conocimientos, habilidades y recursos, así como la pericia de "saber y poder" usarlos (Gaillard et al., 2019; Sandoval Díaz et al., 2018). Específicamente, de acuerdo con Moos y Billings (1982) y Hobfoll (1998), en términos tipológicos, las capacidades de afrontamiento se dividen en dos: (a) activas, que se dirigen al manejo eficaz de las demandas ante una crisis (Gaudreau, 2018); y (b) pasivas, circunscritas a un estilo de desconexión mental o conductual de una situación estresante o psicológicamente exigente (Little, 2018). Ahora bien, dadas las características particulares del riesgo de inundación como evento extremo, de acuerdo con Kellens et al. (2013), cabría distinguir entre acciones protectoras pasivas (mitigación) y acciones protectoras activas (preparación). En este caso, la eficacia en la ejecución de ambas acciones estaría determinada tanto por el nivel de conocimiento como por la eficacia percibida (Amérigo et al., 2019).

En cuarto lugar, en cuanto a sus consecuencias psicológicas, estos eventos han sido conceptualizados como potencialmente traumáticos y desencadenantes de severidad subjetiva, y en algunos casos llevan consigo respuestas desadaptativas como la inacción, el miedo a morir o ser dañado, la falta de control percibido, y la evaluación negativa de respuesta y recuerdos (Beaglehole et al., 2008; García et al., 2013; García et al., 2014; Errázuriz et al., 2019).

Finalmente, si bien estos antecedentes destacan la importancia de estudiar contextualmente (y articular) estos distintos mecanismos psicosociales para afrontar de forma adaptativa los riesgos socionaturales (Amérigo et al., 2019; Berroeta \& Pinto de Carvalho, 2020; OlivosJara et al., 2020; Sapiains \& Ugarte, 2017), es de destacar que para el caso de Chile ha predominado el estudio de amenazas de tipo terremoto y tsunami (Arias et al., 2017; Bronfman et al., 2016; Cid et al., 2012). Para el caso de los aluviones, algunos antecedentes cualitativos destacan el papel del capital social (Castro-Correa et al., 2020), los medios de vida sostenibles familiares (Sandoval-Díaz et al., 2020), y el crecimiento postraumático diferenciado en grupos vulnerados (Sandoval-Díaz \& Cuadra-Martínez, 2020). No obstante, son escasos los estudios nacionales que desarrollan modelos explicativos que articulen factores primarios y secundarios (Lechowska, 2018), y menos en términos de variables mediadoras de las capacidades activas de afrontamiento ante el riesgo aluvional (Navarro et al., $2020 ; 2016)$ en tanto factores centrales para una gestión local y comunicacional efectiva de los riesgos (Birkholz et al., 2014; Lindell \& Perry, 2012).

\section{Para el presente estudio}

El objetivo del presente estudio fue esclarecer la relación entre la experiencia directa (ED), la exposición (EXP) y las capacidades activas de afrontamiento (CAA), considerando tres posibles mediadores: la percepción de riesgo ante aluviones (PRA), la implicancia personal (IP) y la severidad subjetiva (ss) (véase Figura 1).

En particular, el estudio se enfocó en las capacidades de afrontamiento activas, debido a la importancia de la adaptación como un aspecto necesario para la consecución de acciones tanto individuales como colectivas tendientes a la reducción del riesgo de desastre (Gaudreau, 2018; UNDRR, 2015; Wlodarczyk et al., 2016).

Más concretamente, los objetivos específicos del estudio fueron: (a) comparar las CAA, la PRA y la IP entre grupos de experiencia - directa e indirecta - y ubicación — expuestos y no expuestos - ante aluviones; y (b) determinar el grado de asociación e influencia predictiva entre las variables independientes y dependientes implicadas en el análisis mediacional.

Para lograr estos objetivos, se formularon tres hipótesis (véase Figura 1): primero, se esperaba que tanto la ED como la EXP se relacionaran directamente con las CAA (H1) (Kellens et al., 2011; Muñoz-Duque \& Arroyave, 2017); segundo, que la PRA y la IP se relacionaran directamente con las CAA, aunque de mayor manera la PRA (H2) (Navarro et al., 2016; 2020; Raaijmakers et al., 2008) — por el contrario, se esperaba que la ss se relacionara de manera inversa con CAA (Errázuriz et al., 2019; García et al., 2013; 2014; Sandoval-Díaz \& Cuadra-Martínez, 2020), pero en esta línea hay opiniones convergentes respecto a que los factores cognitivos-afectivos de la PR influyen en el despliegue de las CAA de hogares que presentan experiencias directas previas de inundaciones (Kellens et al., 2013; Lechowska, 
Figura 1.

Modelo de mediación múltiple de la experiencia-exposición sobre las capacidades de afrontamiento

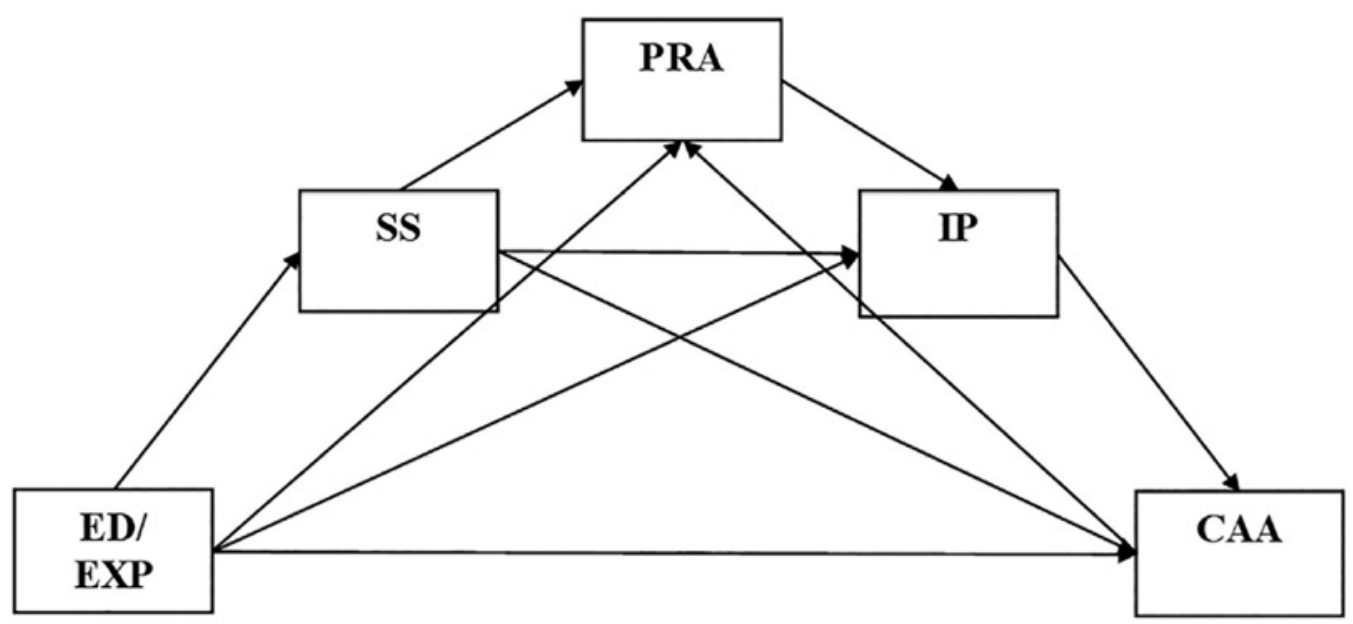

Nota . ED = experiencia directa; $\mathrm{EXP}=$ exposición; $\mathrm{SS}=$ severidad subjetiva; PRA = percepción de riesgo aluvional; $\mathrm{IP}=$ implicancia personal; $\mathrm{CAA}=$ capacidades de afrontamiento activo.

2018); no obstante, la eficacia de este vínculo se ha problematizado, por lo que se sugiere la importancia de otras potenciales variables mediadoras sobre esta relación, como lo son el empoderamiento (Paton et al., 2008), el capital social (Babcicky \& Seebauer, 2017) y la implicancia personal (Navarro et al., 2016; 2020) -; y tercero, que la PRA, la IP y la ss mediaran la relación entre ED/EXP y CAA (H3).

De acuerdo con estos objetivos, analizamos en términos empíricos el caso de los desastres asociados a la amenaza aluvional en el norte chileno.

Los casos: aluviones del norte chileno en 2015 y 2017

Se tuvo en cuenta un evento hidrometeorológico extremo ocurrido entre el 24 y el 26 de marzo de 2015 (25M), producto de una baja segregada asociada a precipitaciones que causó lluvias torrenciales en 17 quebradas de la región de Atacama, Chile. En Copiapó, capital regional, estos sucesos arrastraron gran cantidad de agua, piedras, basura y relaves mineros que enterraron la ciudad bajo una capa de sedimentos de $31 \mathrm{~cm}$ de espesor. En cuanto a la afectación humana, se registraron 22 personas fallecidas, 28000 damnificados, 2000 viviendas destruidas y 5000 con daño mayor, lo que llevó a un impacto económico superior a 46 millones de dólares.

Dos años después, entre el 10 y 13 de mayo de 2017, fuertes lluvias inundaron nuevamente la región, tras lo cual se registraron 5154 damnificados y 204 albergados, así como 221 viviendas destruidas, 1735 con daño mayor y 2258 con daño menor (Cooperativa.cl., 2017, mayo 23). 
por los eventos del 2015 o 2017, y (c) grado de exposición diferenciada ante el riesgo de aluvión, establecido a partir del mapa de las zonas afectadas por el aluvión del 2015 (véase Figura 2).

Tabla 1.

Información descriptiva de los participantes

\begin{tabular}{|c|c|c|c|}
\hline Variable & Pregunta & Categorías & Valor \\
\hline \multirow{2}{*}{ Exposición } & \multirow{2}{*}{ Zona de exposición de la vivienda (Sernageomin, 2015) } & Expuesta al riesgo & $258(52.7 \%)$ \\
\hline & & No expuesta al riesgo & $232(47.3 \%)$ \\
\hline \multirow{2}{*}{ Experiencia } & \multirow{2}{*}{ ¿Su vivienda sufrió daño con el aluvión del 2015 y/o $2017 ?$} & Experiencia directa & $224(45.7 \%)$ \\
\hline & & Experiencia indirecta & $266(54.3 \%)$ \\
\hline \multirow{2}{*}{ Preparación } & \multirow{2}{*}{$\begin{array}{l}\text { ¿Cuentan con un plan de emergencia para afrontar nuevos } \\
\text { aluviones? }\end{array}$} & Sí & $241(49.2 \%)$ \\
\hline & & No & $249(50.8 \%)$ \\
\hline \multirow{2}{*}{ Autoeficacia } & \multirow{2}{*}{ ¿Se siente preparado para afrontar nuevos aluviones? } & Sí & $296(60.4 \%)$ \\
\hline & & No & $194(39.6 \%)$ \\
\hline \multirow{2}{*}{$\begin{array}{l}\text { Confianza } \\
\text { institucional }\end{array}$} & \multirow{2}{*}{$\begin{array}{l}\text { ¿Cree que las autoridades locales están preparadas para } \\
\text { afrontar nuevos aluviones? }\end{array}$} & Sí & $70(14.3 \%)$ \\
\hline & & No & $429(85.7 \%)$ \\
\hline \multirow{2}{*}{ Sexo } & \multirow{2}{*}{ Observada por el/la entrevistador/a } & Femenino & $298(68.8 \%)$ \\
\hline & & Masculino & $192(39.2 \%)$ \\
\hline Edad en años & Autorreporte & & $37.92(D E=17.37)$ \\
\hline $\begin{array}{l}\text { Tiempo } \\
\text { residencial }\end{array}$ & Años de residencia en el sector que vive actualmente & & $15.59(D E=12.30)$ \\
\hline \multirow{3}{*}{ Educación } & \multirow{3}{*}{ Nivel educativo (seleccione la última cursada y aprobada) } & Primaria & $53(10.8 \%)$ \\
\hline & & Secundaria & $282(57.6 \%)$ \\
\hline & & Superior & $155(31.6 \%)$ \\
\hline
\end{tabular}

Figura 2.

Mapa de zonas afectadas por el aluvión (25M)

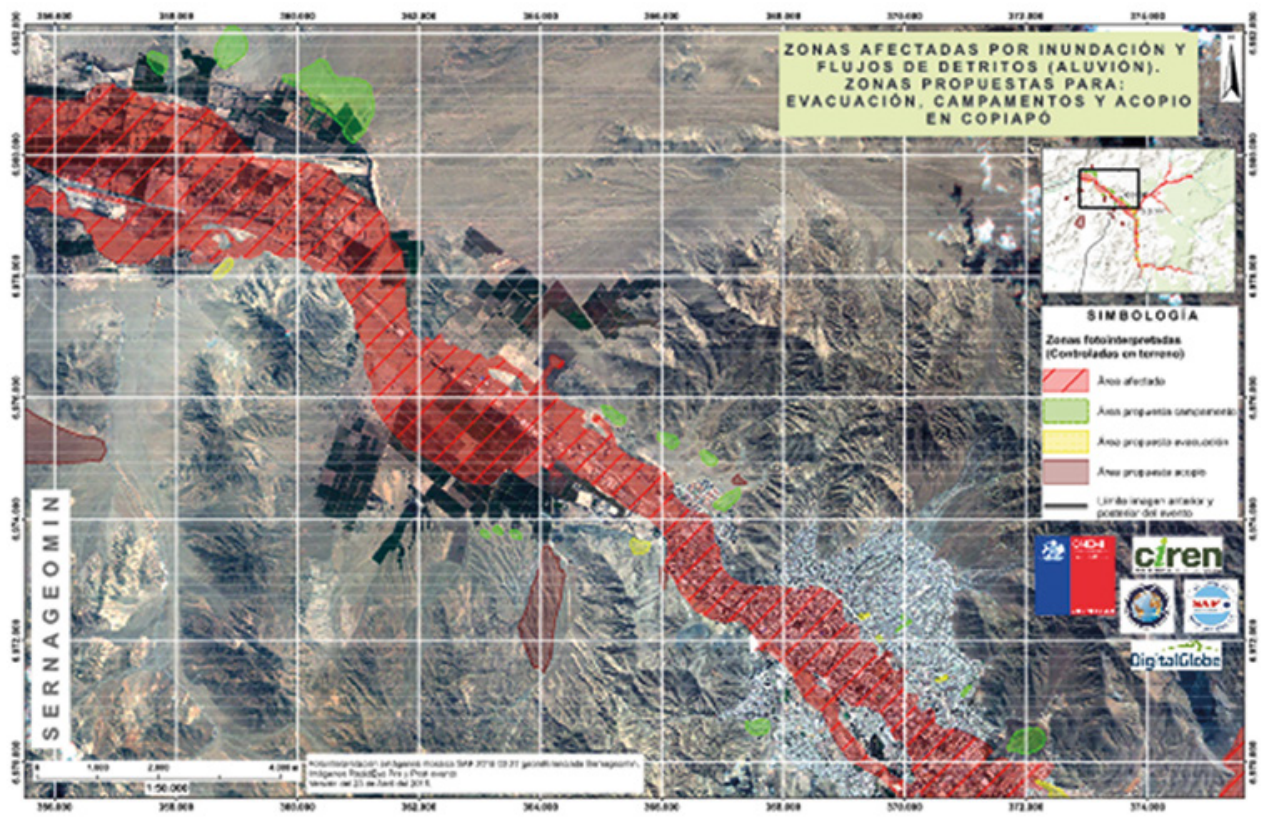

Fuente: Tomado de Servicio Nacional de Geología y Minería de Chile (sernageomin, 2015). 


\section{Instrumentos}

\section{Encuesta sociodemográfica}

Se construyó un instrumento de caracterización sociodemográfica que incluyó preguntas para las variables: (a) edad, (b) sexo, (c) nivel educativo, (d) tiempo de residencia, (e) experiencias previas, (f) preparación, (g) confianza, y (e) zona de habitabilidad en términos de exposición al riesgo (véase Tabla 1). Para esta última variable, se utilizó el mapa de zonas afectadas por inundación y flujo de detritos desarrollado por el Servicio Nacional de Geología y Minería de Chile (SERnageomin, 2015, véase Figura 2). Con el objetivo de utilizar un formato estándar para las variables mencionadas, utilizamos las preguntas $-\mathrm{y}$ opciones de respuesta - de la encuesta de caracterización socioeconómica post-terremoto 2010 (Ministerio de Desarrollo Social y Familia de Chile, 2010).

\section{Escala de Percepción del Riesgo de Inundación}

Esta escala, desarrollada por Navarro et al. (2016), es una adaptación de la versión de Terpstra et al. (2005) que, en su versión original, consta de ocho factores y 23 ítems (confiabilidad entre $\alpha=.54$ y $\alpha=.82$, varianza total explicada del 74 \%). La versión adaptada por Navarro et al. (2016) presenta 14 ítems con opciones de respuesta que van de 0 ("muy en desacuerdo") a 4 ("muy de acuerdo"), y cuenta con tres factores: (a) conocimiento del riesgo, con seis ítems, (b) temor-afectación, con tres ítems, y (c) aumento global del riesgo, con cinco ítems (confiabilidad entre $\alpha=.63 \mathrm{y}$ $\alpha=.79$, varianza total explicada del $58.78 \%$ ).

Como método de validación del constructo, se realizó un análisis factorial confirmatorio (AFC) utilizando como método de estimación el de mínimos cuadrados diagonalmente ponderados (DWLS), adecuado para variables categóricas con matrices policóricas (Newsom, 2018). El AFC presentó un nivel de ajuste adecuado para el modelo de tres factores $\left(x^{2}=1553.313(p=0.07)\right.$; RMSEA $=0.023$ $(\mathrm{IC} 95 \%=0.000-0.036) ; \mathrm{TLI}=0.930 ; \mathrm{CFI}=0.943)$. La fiabilidad de la subescala de conocimiento del riesgo fue de $\omega=0.72$, para temor-afectación fue de $\omega=0.60$, y para aumento global del riesgo, de $\omega=0.71$.

\section{Escala de Capacidades de Afrontamiento}

Esta escala, desarrollada por Navarro et al. (2016), es una adaptación al riesgo de inundación de la versión validada de López-Vázquez y Marván (2004), que en su versión original cuenta con 26 ítems (confiabilidad entre $\alpha=.77$ y $\alpha=.83$, varianza total explicada del $31.64 \%$ ). La versión adaptada por Navarro et al. (2016) presenta 15 ítems con opciones de respuesta que van de 0 ("muy en desacuerdo") a 4 ("muy de acuerdo"), y dos factores de afrontamiento: (a) activo, con once ítems, y (b) pasivo, con cuatro ítems (confiabilidad entre $\alpha=.72$ y $\alpha=.89$, varianza total explicada del $46 \%$ ).

Para el presente estudio, el AFC presentó un ajuste moderado respecto al modelo de dos factores $\left(x^{2}=5230.60\right.$ $(p=0.00) ;$ RMSEA $=0.059$ (IC $95 \%=0.050-0.068)$; $\mathrm{TLI}=0.966 ; \mathrm{CFI}=0.971)$. La fiabilidad de la escala total fue de $\omega=0.85$, mientras que para la subescala de afrontamiento activo fue de $\omega=0.89$, y en afrontamiento pasivo, de $\omega=0.55$.

\section{Escala de Implicación Personal}

Desarrollada por Navarro et al. (2016), es una adaptación lingüística y temática realizada a partir de la versión de Demarque et al. (2011), con siete ítems con opciones de respuesta que van de 0 ("nunca") a 4 ("siempre"), y dos factores: (a) identificación y posibilidad percibida de acción, que refiere al grado de identificación y sentimiento de eficacia personal, con cinco ítems; y (b) valoración, es decir, el grado de importancia del problema, con dos ítems. La prueba cuenta con una confiabilidad entre $\alpha=.66$ y $\alpha=.75$, y una varianza total explicada del $68.86 \%$.

Para el presente estudio, el AFC presentó un ajuste moderado respecto al modelo de dos factores $\left(x^{2}=1082.92\right.$, $p=0.02 ;$ RMSEA $=0.045($ IC $95 \%=0.018-0.070) ; \mathrm{TLI}=0.981$; $\mathrm{CFI}=0.988$ ). La fiabilidad de la escala total fue de $\omega=0.80$, mientras que para la subescala de identificación y posibilidad percibida de acción fue de $\omega=0.79$, y en valoración, de $\omega=0.78$.

\section{Escala de Severidad Subjetiva del Evento}

Elaborada por García et al. (2013), consta de dos ítems que miden si la persona percibe que el evento ha alterado su vida, y si lo percibe como traumático. Cada pregunta fue respondida en una escala Likert de 5 puntos, con un rango de 0 a 8 puntos. La fiabilidad total de la versión utilizada con estudiantes universitarios expuestos a un terremoto contó con un $\alpha=.69$. Para el presente estudio, la fiabilidad de la escala fue de $\alpha=.80$.

\section{Análisis de datos}

En primer lugar, se realizó una comparación de las variables estudiadas entre los grupos de diferente ubicación (no expuestos/expuestos) y experiencia (directa/indirecta). Para las diferencias entre grupos, se utilizó la prueba de $t$ de Student para muestras independientes, y se determinó el tamaño del efecto $d$ y la potencia estadística 1- $\beta$.

En segundo lugar, antes de realizar el análisis mediacional, analizamos el grado de asociación correlacional 
de Pearson entre variables, para posteriormente utilizar el análisis de regresión lineal múltiple por medio del método por pasos sucesivos. Para analizar el papel mediador múltiple, se utilizó el modelo seis y el procedimiento descrito por Preacher y Hayes (2008), el cual permite el análisis simultáneo y la comprobación estadística de cada uno de los efectos indirectos estimados - mediación - en un modelo a través de cada mediador y los efectos directos de la variable predictora sobre la variable dependiente. Además, se utilizó el procedimiento bootstrapping con 10000 repeticiones, ya que tiene mayor control del error Tipo I que el enfoque tradicional de la regresión múltiple de tres pasos (Baron \& Kenny, 1986) o la prueba de Sobel (Sobel, 1982) para la estimación de la mediación y efectos indirectos.

En particular, el bootstrapping es una estrategia de remuestreo para la estimación y la prueba de hipótesis. Con el método de arranque, la muestra se conceptualiza como una pseudopoblación que representa la población más amplia de la muestra original. Este tipo de análisis determina si el efecto indirecto de los mediadores es significativamente diferente de cero a través de los intervalos de confianza del $95 \%$. Si para cada efecto indirecto el intervalo de confianza no incluye el 0 , entonces el efecto indirecto es significativo $(p<.05) \mathrm{y}$, por tanto, se puede afirmar que la mediación existe.

Finalmente, para el procesamiento estadístico de datos se utilizó el software sPss 22.0, mientras que para la mediación moderada se utilizó la macro PROCESS de SPSS, proporcionada por Preacher y Hayes (2008).

\section{Resultados}

Comparación de medias y tamaño del efecto de las variables estudiadas entre exposición y experiencia ante al riesgo aluvional

Previo a la realización de la prueba $t$, se verificó el cumplimiento de los supuestos de: (a) independencia, pues las observaciones de los grupos no se encuentran relacionadas; (b) normalidad, ya que el tamaño muestral está por encima de 40 observaciones; y (c) homocedasticidad, con la Prueba de Levene, incorporada en las Tablas 2 y 3 (Pardo et al., 2014).

En la Tabla 2 se observan los resultados obtenidos para los grupos expuestos y no expuestos, donde se identifican las siguientes diferencias significativas y tamaños del efecto: (a) grande, en severidad subjetiva, (b) moderado, en afrontamiento activo; y (c) pequeños, en identificación y posibilidad percibida de acción, valoración, temor al riesgo, y afrontamiento pasivo.

Para los grupos de experiencia directa e indirecta, se identificaron diferencias significativas en las mismas variables que en los grupos expuestos y no expuestos, así como también tamaños de efecto similares (véase Tabla 3 ).

\section{Asociación entre las variables estudiadas}

Previo a la realización de la prueba de correlación de Pearson, se verificó el cumplimiento del supuesto de normalidad univariante por medio de la revisión del grafico

Tabla 2.

Variables estudiadas según exposición al riesgo de inundación

\begin{tabular}{|c|c|c|c|c|c|c|c|c|c|c|}
\hline & \multirow[t]{2}{*}{ Levene $(F)$} & \multicolumn{2}{|c|}{$\begin{array}{c}\text { No expuestos al } \\
\text { riesgo }\end{array}$} & \multicolumn{2}{|c|}{ Expuestos al riesgo } & \multirow[t]{2}{*}{ IC $95 \%$} & \multirow[t]{2}{*}{$t$} & \multirow[t]{2}{*}{$\alpha$} & \multirow[t]{2}{*}{$d$} & \multirow[t]{2}{*}{$1-\beta$} \\
\hline & & $M$ & $D E$ & $M$ & $D E$ & & & & & \\
\hline PRA total & 0.69 & 33.35 & 6.71 & 34.03 & 6.44 & {$[-1.85 / 0.48]$} & -1.15 & .250 & -.10 & .54 \\
\hline PRA temor & 0.19 & 7.40 & 2.80 & 8.36 & 2.75 & {$[-1.46 /-0.47]$} & $-3.85 * *$ & .000 & -.35 & .78 \\
\hline PRA conocimiento & 0.15 & 8.52 & 4.41 & 7.75 & 4.39 & {$[-0.01 / 1.55]$} & 1.93 & .540 & .17 & .93 \\
\hline PRA aumento global & 0.03 & 14.19 & 3.33 & 14.52 & 3.28 & {$[-0.92 / 0.25]$} & -1.12 & .264 & -.10 & .53 \\
\hline IP total & 2.42 & 17.49 & 5.26 & 19.67 & 4.70 & {$[-3.06 /-1.30]$} & $-4.84 * *$ & .000 & -.44 & .93 \\
\hline IP identificación & 0.03 & 11.07 & 4.12 & 12.84 & 4.02 & {$[-2.49 /-1.04]$} & $-4.80 * *$ & .000 & -.43 & .93 \\
\hline IP valoración & $8.63 *$ & 6.35 & 1.89 & 6.83 & 1.43 & {$[-0.78 /-0.18]$} & $-3.14 *$ & .002 & -.29 & .80 \\
\hline CA total & 0.81 & 22.72 & 10.34 & 28.72 & 11.02 & {$[-7.90 /-4.09]$} & $-6.19 * *$ & .000 & -.56 & .99 \\
\hline CA activo & 0.15 & 17.70 & 9.34 & 22.72 & 9.60 & {$[-6.72 /-3.34]$} & $-5.86 * *$ & .000 & -.53 & .99 \\
\hline CA pasivo & 3.11 & 5.03 & 3.14 & 6.00 & 3.52 & {$[-1.56 /-0.37]$} & $-3.19 *$ & .002 & -.29 & .54 \\
\hline Severidad subjetiva & 1.86 & 3.07 & 2.06 & 4.93 & 2.13 & {$[-2.23 /-1.49]$} & $-9.82 * *$ & .000 & -.89 & 1 \\
\hline
\end{tabular}

Nota. PRA = percepción riesgo aluvional; $\mathrm{IP}=$ implicancia personal; $\mathrm{CA}=$ capacidades de afrontamiento. ${ }^{*} p<.05$ (bilateral). ${ }^{* *} p<.01$ (bilateral). 
Tabla 3.

Variables estudiadas según experiencia

\begin{tabular}{|c|c|c|c|c|c|c|c|c|c|c|}
\hline & \multirow{2}{*}{$\begin{array}{l}\text { Levene } \\
\text { (F) }\end{array}$} & \multicolumn{2}{|c|}{$\begin{array}{c}\text { Experiencia } \\
\text { indirecta }\end{array}$} & \multicolumn{2}{|c|}{$\begin{array}{c}\text { Experiencia } \\
\text { directa }\end{array}$} & \multirow[t]{2}{*}{ IC $95 \%$} & \multirow[t]{2}{*}{$t$} & \multirow[t]{2}{*}{$\alpha$} & \multirow[t]{2}{*}{$d$} & \multirow[t]{2}{*}{$1-\beta$} \\
\hline & & $M$ & $D E$ & $M$ & $D E$ & & & & & \\
\hline PRA total & 0.41 & 33.32 & 6.66 & 34.16 & 6.46 & {$[-2.00 / 0.33]$} & -1.41 & .160 & -.13 & .50 \\
\hline PRA temor & 0.05 & 7.45 & 2.78 & 8.45 & 2.77 & {$[-1.49 /-0.50]$} & $-3.96 * *$ & .000 & -.36 & .74 \\
\hline PRA conocimiento & 0.00 & 8.34 & 4.37 & 7.84 & 4.46 & {$[-0.28 / 1.29]$} & 1.26 & .210 & .12 & .51 \\
\hline PRA aumento global & 0.04 & 14.27 & 3.35 & 14.47 & 3.35 & {$[-0.80 / 0.38]$} & -0.69 & .492 & -.06 & .58 \\
\hline IP total & 0.28 & 17.76 & 5.15 & 19.68 & 4.82 & {$[-2.81 /-1.02]$} & $-4.22 * *$ & .000 & -.39 & .82 \\
\hline IP identificación & 0.26 & 11.24 & 4.05 & 12.91 & 4.11 & {$[-2.39 /-0.94]$} & $-4.51 * *$ & .000 & -.41 & .88 \\
\hline IP valoración & $4.67 *$ & 6.47 & 1.83 & 6.77 & 1.46 & {$[-0.60 /-0.01]$} & $-2.06 *$ & .040 & -.18 & .99 \\
\hline CA total & 0.09 & 22.76 & 10.55 & 29.58 & 10.63 & {$[-8.72 /-4.94]$} & $-7.12 * *$ & .000 & -.64 & .99 \\
\hline CA activo & 0.60 & 17.64 & 9.52 & 23.54 & 9.17 & {$[-7.67 /-4.24]$} & $-6.95 * *$ & .000 & -.63 & .99 \\
\hline CA pasivo & 2.08 & 5.12 & 3.18 & 6.04 & 3.55 & {$[-1.52 /-0.32]$} & $-3.19 *$ & .003 & -.27 & .51 \\
\hline Severidad subjetiva & 1.17 & 3.16 & 2.06 & 5.11 & 2.11 & {$[-2.32 /-1.58]$} & $-10.34 * *$ & .000 & -.94 & 1 \\
\hline
\end{tabular}

Nota. PRA = percepción riesgo aluvional; $\mathrm{IP}=$ implicancia personal; $\mathrm{CA}=$ capacidades de afrontamiento. $* p<.05($ bilateral). $* * p<.01$ (bilateral).

Tabla 4.

Matriz de correlaciones entre las variables de estudio

\begin{tabular}{|c|c|c|c|c|c|c|c|c|}
\hline & 1. & 2. & 3. & 4. & 5. & 6. & 7. & 8. \\
\hline 1. PRA temor & 1 & & & & & & & \\
\hline 2. PRA conocimiento & $.09 *$ & 1 & & & & & & \\
\hline 3. PRA aumento global & $.36 * *$ & $.18 * *$ & 1 & & & & & \\
\hline 4. IP identificación & $.45 * *$ & .01 & $.40 * *$ & 1 & & & & \\
\hline 5. IP valoración & $.26 * *$ & -.09 & $.36 * *$ & $.39 * *$ & 1 & & & \\
\hline 6. CA activo & $.31 * *$ & $.15 * *$ & $.26 * *$ & $.49 * *$ & $.16 * *$ & 1 & & \\
\hline 7. CA pasivo & -.03 & .07 & .05 & $.13 * *$ & .01 & $.24 * *$ & 1 & \\
\hline 8. Severidad subjetiva & $.46 * *$ & $-.12 *$ & $.33 *$ & $.53 * *$ & $.26 * *$ & $.11 *$ & $.11 *$ & 1 \\
\hline
\end{tabular}

Nota. $N=490 ;$ PRA = percepción riesgo aluvional; $\mathrm{IP}=$ implicancia personal; $\mathrm{CA}=$ capacidades de afrontamiento. ${ }^{*} p<.05$ (bilateral).

$* * p<.01$ (bilateral).

Tabla 5.

Regresión sobre capacidades de afrontamiento activas

\begin{tabular}{cccccccccc}
\hline Modelo 5 & $\mathrm{F}(\mathrm{gl})$ & $\mathrm{R} 2$ & $\Delta \mathrm{R} 2$ & $\mathrm{~B}$ & $\begin{array}{c}\text { Error } \\
\text { estándar }\end{array}$ & $\beta$ & $p$ & $1-\beta$ & $\mathrm{f} 2$ \\
\hline IP identificación & & & & 0.904 & .093 & .383 & .000 & \\
*Preparación & 58.703 & \multirow{2}{*}{.422} & .415 & 3.434 & .728 & .175 & .000 & 1 & .730 \\
*Experiencia & $(6.483)$ & & & 3.197 & .775 & .160 & .000 & & \\
*Autoeficacia & & & & 0.503 & .139 & .114 & .000 & \\
PRA temor & & & & 4.776 & .741 & .244 & .000 & \\
\hline
\end{tabular}

Nota. IP $=$ implicancia personal; PRA $=$ percepción de riesgo aluvional. $*$ Variable dummy.

de histograma y el cálculo del Z de simetría (valores entre -1.61 y 0.06 ) y $Z$ de curtosis (valores entre 1.97 y -0.16 ).

En la Tabla 4 se presenta el grado de asociación entre las variables, donde se enfatiza la asociación de afrontamiento activo con una intensidad de relación: (a) media, en identificación y posibilidad percibida de acción, y temor al riesgo; y (b) pequeña, en conocimiento, aumento global del riesgo, valoración, afrontamiento pasivo, y severidad subjetiva.
Regresión sobre la capacidad activa de afrontamiento

Para saber si la CAA está predicha por las dimensiones de PRA, IP, SS y las características/condiciones sociodemográficas - las variables categóricas se reconvirtieron en variables indicadores (dummy) - se llevó a cabo una regresión múltiple. Los resultados, que se observan en la Tabla 5, muestran que todos los índices de significancia, potencia estadística y tamaño del efecto son adecuados, de modo que predicen en conjunto el $42.2 \%$ de las capacidades activas de afrontamiento. 
En cuanto al cumplimiento del supuesto, para los indicadores de multicolinealidad VIF se obtuvo valores inferiores a 10 [1.053-1.327], y para Tolerancia, valores mayores a .20 [.753-.950]. Por último, el indicador de Durbin Watson se ubicó dentro de las dos unidades, lo que permite una generalización de los datos.

Por tanto, los resultados obtenidos del análisis de regresión por pasos sucesivos muestran que el modelo de cinco predictores presenta un nivel de potencia estadística $(1-\beta=1)$ y tamaño de efecto $\left(f^{2}=.730\right)$ relevantes para la predicción de las CAA.

\section{Análisis de mediación múltiple}

El diagrama de rutas correspondiente al modelo de mediación de las CAA se presenta en la Figura 3, donde se incluyen los pesos estandarizados de los efectos directos y la varianza explicada de las variables. Como se puede observar en el diagrama, la relación entre la ED y las CAA estaría mediada por el temor-afectación (PRA), la identificación y la posibilidad percibida de acción (IP) de forma significativa. Los parámetros no estandarizados y estandarizados del modelo de mediación se muestran en la Tabla 4, así como sus intervalos de confianza al $95 \%$.

De acuerdo con la Tabla 6, los efectos indirectos estandarizados $\mathrm{ED} \rightarrow \mathrm{IP} \rightarrow$ CAA y ED $\rightarrow$ PRA $\rightarrow$ IP $\rightarrow$ CAA se podrían categorizar como un efecto aproximadamente pequeño; resultados que indican que las variables de PRA e IP mediaron la relación entre la ED y las capacidades activas. El efecto indirecto restante de la $\mathrm{ED}(\mathrm{ED} \rightarrow \mathrm{PRA} \rightarrow \mathrm{CAA})$ no fue significativo, lo cual indica que la PRA sola no media la relación entre el ED y las CAA.

Figura 3.

Modelo estandarizado de mediación múltiple de las capacidades activas de afrontamiento

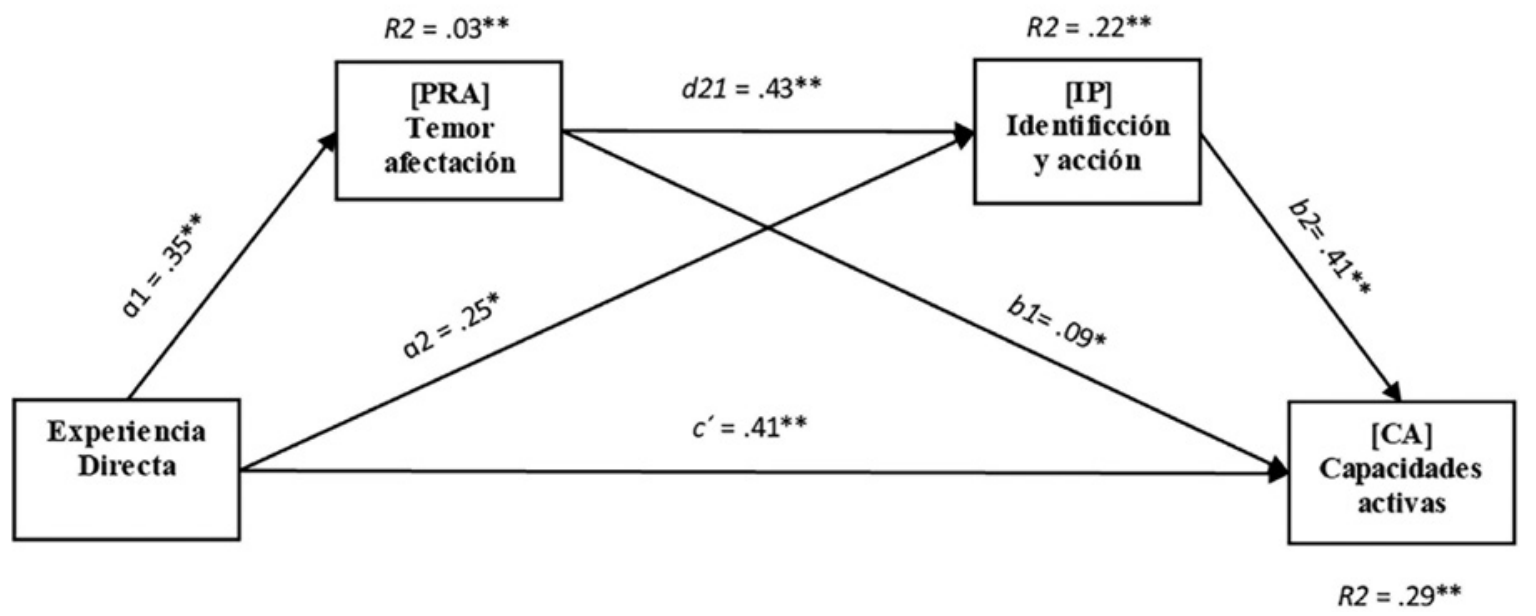

Nota. $R 2$ = varianza explicada. Las flechas unidireccionales completas denotan regresiones significativas. ${ }^{*} p<.05 .{ }^{* *} p<.01$.

Tabla 6.

Parámetros estimados del modelo de mediación de capacidades activas

\begin{tabular}{|c|c|c|c|c|}
\hline \multirow{2}{*}{ Tipo de efecto/Efecto } & \multicolumn{2}{|c|}{ No estandarizados } & \multicolumn{2}{|c|}{ Estandarizados } \\
\hline & Valor estimado & $95 \% \mathrm{IC}$ & Valor estimado & $95 \%$ IC \\
\hline \multicolumn{5}{|l|}{ Efecto de ED a CAa } \\
\hline Total & $5.905 * *$ & $4.237,7.574$ & $0.602 * *$ & \\
\hline Total indirectos & $1.982 * *$ & $1.086,2.819$ & $0.197 * *$ & $0.112,0.283$ \\
\hline \multicolumn{5}{|l|}{ Específicos indirectos } \\
\hline $\mathrm{ED} \rightarrow \mathrm{TA} \rightarrow \mathrm{Caa}$ & 0.308 & $-0.002,0.710$ & 0.031 & $-0.000,0.072$ \\
\hline $\mathrm{ED} \rightarrow \mathrm{IPA} \rightarrow \mathrm{CAa}$ & $1.007 * *$ & $0.350,1.697$ & $0.1028 * *$ & $0.036,0.172$ \\
\hline $\begin{array}{c}\mathrm{ED} \rightarrow \mathrm{TA} \rightarrow \mathrm{IP} \rightarrow \mathrm{CAa} \\
\text { Directo }\end{array}$ & $0.613 * *$ & $0.280,1.016$ & $0.062 * *$ & $0.029,0.103$ \\
\hline $\mathrm{ED} \rightarrow \mathrm{CAA}$ & $3.977 * *$ & $2.463,5.491$ & $0.406 * *$ & \\
\hline
\end{tabular}

Nota ED = experiencia directa; $\mathrm{TA}=$ temor-afectación sobre el riesgo; $\mathrm{IPA}=$ identificación y posibilidad percibida de acción; $\mathrm{CAA}=\mathrm{ca}-$ pacidades activas de afrontamiento. La variable experiencia se codificó en indirecta $=0$ y directa $=1 .{ }^{*} p<.05,{ }^{* *} p<.01$. 


\section{Discusión}

Como primer resultado, tanto las personas expuestas como las que poseen experiencia directa previa con aluviones presentaron una alta percepción del riesgo basada en el temor e implicancia personal, a diferencia de quienes poseen experiencias indirectas o sin exposición (Muñoz-Duque \& Arroyave, 2017; Navarro et al., 2020; 2016). Al igual que estudios previos con población no experta, las personas con experiencia o exposición tendieron a percibir el riesgo por medio del temor-afectación - más que por el conocimiento del riesgo en sí-, emoción que podría explicarse por la sensación de vulnerabilidad previa (Botzen et al., 2009; Ho et al., 2008; Slovic, 2000; Zapa-Pérez et al., 2017).

A su vez, el potencial despliegue de acciones de afrontamiento requirió de la combinación de esta emoción y la autoeficacia, en términos de la valorización adecuada de la amenaza y la identificación y posibilidad de acción (Grothmann \& Reusswig, 2006; Tannenbaum et al., 2015). No obstante, varios estudios resaltan la importancia predictiva de la eficacia grupal del capital social sobre la autoeficacia; esto debido al mayor control percibido de la acción colectiva sobre situaciones de riesgo natural o vulnerabilidad estructural (Bostrom et al., 2018; Babcicky \& Seebauer, 2017).

A estos antecedentes se suma el impacto subjetivo tanto en términos de severidad traumática como de alteración vital de las inundaciones vivenciadas en 2015 y 2017 respectivamente (Beaglehole et al., 2008; García et al., 2013; García et al., 2014; Sandoval-Díaz \& Cuadra-Martínez, 2020; Wlodarczyk et al., 2016), de manera que es posible identificar el papel central de las emociones de valencia negativa como amplificadores perceptivos y motivadores de la conducta (Tompkins et al., 2018).

Por último, si bien ambas capacidades de afrontamiento son significativas en población expuesta y con experiencia directa, la disposición de tácticas activas predomina respecto a las pasivas, lo cual podría vincularse a la experiencia directa, ya que conlleva no solo la expresión latente de las emociones mencionadas, sino también la adquisición informal de conocimiento práctico, los esquemas de afrontamiento y la autopercepción de control - y preparaciónante potenciales riesgos hidrometereológicos (Fox et al., 2017; Gaillard et al., 2019; Guillard et al., 2019; Kellens et al., 2013; Wachinger et al., 2013).

\section{Conclusiones}

Respecto al objetivo central del estudio, es decir, la evaluación de las posibles variables mediadoras de la relación entre la experiencia directa y las capacidades activas, los resultados indican que: (a) el temor-afectación media la relación entre la ED y las CAA (efecto pequeño), (b) la IP media la relación entre la ED y las CAA (efecto mediano); y (c) los efectos directos de la ED hacia las CAA disminuyen significativamente al considerar el proceso de mediación completo.

Estos hallazgos indican que la experiencia directa, en congruencia con las expectativas del estudio, funciona como un heurístico de disponibilidad (Siegrist \& Gutscher, 2006), puesto que predispone a una mayor percepción "afectiva" y a implicarse activamente para afrontar potenciales situaciones de riesgo. En esta línea, metaanálisis recientes concluyen que emociones de valencia negativa (miedo e ira) y la autoeficacia percibida impulsarían actitudes y comportamientos precautorios (Ruiter et al., 2014; Tannenbaum et al., 2015). En este caso, el despliegue de las capacidades de afrontamiento se vería motivado por el potencial implicativo de acción, lo que amplificaría la percepción del riesgo a través del miedo vivenciado tras el desastre aluvional de 2015 (Ruiter et al., 2014; Tompkins et al., 2018).

Por otra parte, en el caso de la ss, y contrario a las hipótesis planteadas, se encontró que esta variable no ejerce un rol mediador respecto de la CAA; resultado que sugiere que la relación entre la ss y las CAA podría estar explicada por aquellas características que comparte con el TA - en términos de emociones de valencia negativa - o por otros aspectos que deben ser investigados con posterioridad.

Finalmente, respecto a las limitaciones del estudio, cabe destacar las debilidades del ordenamiento temporal del diseño correlacional-transversal, pues este no puede verificarse con certeza. A su vez, dado que la mediación implica el producto de dos coeficientes de trayectoria causal, este resultado es especialmente sensible a las fluctuaciones muestrales, por lo cual debe revisarse su generalidad frente a otros riesgos socionaturales. Posteriores investigaciones deben complejizar el modelo propuesto mediante SEM, al incorporar medidas robustas de las variables dummy significativas en la regresión (autoeficacia y preparación).

En línea con Wachinger et al. (2013), Lechowska (2018), Bronfman et al. (2016) y Raaijmakers et al. (2008), proponemos ampliar el modelo propuesto con mecanismos psicosociales como (a) autoeficacia - ya que en el presente estudio el $60.4 \%$ de los participantes se siente preparado para afrontar nuevos aluviones-, (b) confianza en las autoridades/expertos — pues el $85.7 \%$ de los participantes cree que las autoridades no están preparadas para enfrentar potenciales inundaciones - y (c) preparación - porque el $50.8 \%$ todavía no cuenta con un plan de emergencia familiar-, en pos de una gestión constructivista del riesgo 
140

(Birkholz et al., 2014) y efectiva comunicacionalmente (Lindell \& Perry, 2012).

Otros elementos interpersonales y contextuales para considerar son el capital social (Babcicky \& Seebauer, 2017; Castro-Correa et al., 2020), la resiliencia comunitaria (Adger \& Brown, 2009, Sandoval-Díaz, 2020), el apego de lugar (Berroeta, \& Pinto de Carvalho, 2020; Guillard et al., 2019), el empoderamiento (Paton et al., 2008) y la propiedad de la vivienda (Grothmann \& Reusswig, 2006), los cuales deberían sumarse a los de carácter intrapersonal - características de vulnerabilidad socioeconómica, edad, educación, sexo- (Botzen et al., 2009; Cid et al., 2012; Kellens et al., 2011; 2013) y a las dimensiones disposicionales de ajuste psicológico, como la reevaluación, la expresión emocional regulada, el autocontrol no represivo y la descarga emocional (Olivos-Jara et al., 2020; Sapiains \& Ugarte, 2017; Wlodarczyk et al., 2016). En síntesis, son necesarios más estudios sobre la comprensión de respuestas adaptativas-resilientes ante la intensificación riesgos hidrometereológicos bajo el contexto del cambio climático (Amérigo et al., 2019; IPCC, 2014).

\section{Referencias}

Adger, W., \& Brown, K. (2009). Vulnerability and resilience to environmental change: ecological and social perspectives. En N. Castree, D. Demeritt, D. Liverman y B. Rhoads (Eds.), A companion to environmental geography (pp. 109-122). Blackwell Publishing Ltd. https://doi. org/10.1002/9781444305722.ch8

Amérigo, M., García, J. A., Pérez-López, R., \& Talayero, F. (2019). Retos para afrontar el cambio climático: análisis de la percepción del riesgo de inundación ante riadas. Papeles del psicólogo, 40(3), 197-204. https://doi.org/10.23923/ pap.psicol2019.2903

Arias, J., Bronfman, N., Cisternas, P., \& Repetto, P. (2017). Hazard proximity and risk perception of tsunamis in coastal cities: ¿Are people able to identify their risk? Plos one, 12(10), 1-13. https://doi.org/10.1371/journal.pone.0186455

Babcicky, P., \& Seebauer, S. (2017). The two faces of social capital in private flood mitigation: opposing effects on risk perception, self-efficacy and coping capacity. Journal of Risk Research, 20(8), 1017-1037. https://doi.org/10.1080/1 3669877.2016.1147489

Baron, R. M., \& Kenny, D. A. (1986). The moderator-mediator variable distinction in social psychological research: Conceptual, strategic, and statistical considerations. Journal of Personality and Social Psychology, 51(6), 1173-1182. https://doi.org/10.1037/0022-3514.51.6.1173

Beaglehole, B., Mulder, R., Frampton, C., Boden, J., NewtonHowes, G., \& Bell, C. (2018). Psychological distress and psychiatric disorder after natural disasters: systematic review and meta-analysis. The British Journal of Psychiatry, 213(6), 716-722. https://doi.org/10.1192/bjp.2018.210

Berroeta, H., \& Pinto de Carvalho, L. (2020). La Psicología Ambiental-Comunitaria en el estudio de los desastres: la importancia de los vínculos socioespaciales. Psykhe, 29(1), 1-16. http://dx.doi.org/10.7764/psykhe.29.1.1579

Birkholz, S., Muro, M., Jeffrey, P., \& Smith, H. M. (2014). Rethinking the relationship between flood risk perception and flood management. Science of the Total Environment, 478, 12-20. https://doi.org/10.1016/j.scitotenv.2014.01.061

Bodemer, N., \& Gaissmaier, W. (2015). Risk Perception. En H. Cho, T. Reimer, y K. A. McComas (Eds.). The SAGE Handbook of Risk Communication (pp. 10-23). Sage.

Bostrom, A., Böhm, G., \& O’Connor, R. (2018). Communicating Risks: Principles and Challenges. En M. Raue, E. Lermer y B. Streicher (Eds.), Psychological Perspectives on Risk and Risk Analysis: Theory, Models, and Applications (pp. 251278). Springer.

Botzen, W., Aerts, J. \& van den Bergh, J. (2009). Dependence of flood risk perceptions on socioeconomic and objective risk factors. Water Resources Research, 45(10), 1-15. https://doi.org/10.1029/2009WR007743

Bronfman, N., Cisternas, P., López-Vázquez, E., \& Cifuentes, L. (2016). Trust and risk perception of natural hazards: implications for risk preparedness in Chile. Natural Hazards, 81(1), 307-327. https://doi.org/10.1007/s11069-015-2080-4

Burkett V. (2013). Coping Capacity. En P. Bobrowsky (Ed.), Encyclopedia of Natural Hazards. Encyclopedia of Earth Sciences Series (pp. 119-121). Springer. https://doi. org/10.1007/978-1-4020-4399-4_75

Castro-Correa, C., Aldunce, P., Wyndham, K., Mena, D., \& Pérez, S. (2020). Transformation of social capital during and after a disaster event: the cases Chañaral and Diego de Almagro, Atacama Region, Chile. Natural Hazards, 103(2), 2427-2440. https://doi.org/10.1007/s11069-020-04091-9

Cid, G., Castro, C., \& Rugiero de Souza, V. (2012). Percepción del riesgo en relación con capacidades de autoprotección y autogestión, como elementos relevantes en la reducción de la vulnerabilidad en la ciudad de La Serena. Revista INVI, 27(75), 105-142. http://dx.doi.org/10.4067/ S0718-83582012000200004

Cooperativa.cl. (23 de mayo 2017). 2 muertos y más de 5 mil damnificados tras sistema frontal en el norte del país. http:// www.cooperativa.cl/noticias/pais/region-de-atacama/2muertos-y-mas-de-5-mil-damnificados-tras-sistema-fron tal-en-el-norte/2017-05-22/234921.html

Demarque, C., Lo Monaco, G., Apostolidis, T., \& Guimelli, C. (2011). Socialisation, perspectives temporelles et implication personnelle: une étude dans le champ de l'environnement. Les Cahiers Internationaux de Psychologie Sociale, 92(4), 351-369. https://doi.org/10.3917/cips.092.0351

Dzialek, J. (2013). Perception of Natural Hazards and Disasters. En P. Bobrowsky (Ed.), Encyclopedia of Natural Hazards. 
Encyclopedia of Earth Sciences Series (pp. 756-759). Springer. https://doi.org/10.1007/978-1-4020-4399-4_265

Errázuriz, P., Fischer, C., Behn, A., Letelier, C., \& Monari, M. (2019). Orientaciones prácticas para psicoterapeutas que atienden a pacientes con TEPT después de un desastre natural. Psykhe (Santiago), 28(1), 1-13. https://dx.doi. org/10.7764/psykhe.28.1.1218

Flament, C., \& Rouquette, M. L. (2003). Anatomie des idées ordinaires. Comment étudier les représentations sociales. Armand Colin.

Fox, K., Lauve-Moon, K., \& Powers, B. (2017). Risk and recovery: Understanding flood risk perceptions in a postdisaster city-The case of New Orleans. Sociological spectrum, 37(6), 335-352. https://doi.org/10.1080/02732173.2017.13 65029

França-Tarragó, O. (1996). Los dilemas éticos de la práctica psicológica y psiquiátrica. Revista de psicoterapia, 7(25), 39-50. https://dialnet.unirioja.es/servlet/ articulo? codigo $=2968268$

Gaillard, J., Cadag, J., \& Rampengan, M. (2019). People's capacities in facing hazards and disasters: an overview. Natural Hazards, 95(3), 863-876. https://doi.org/10.1007/ s11069-018-3519-1

García, F., Cova, F., \& Reyes, A. (2013). Severidad del trauma, optimismo, crecimiento postraumático y bienestar en sobrevivientes de un desastre natural. Universitas Psychologica, 13(2), 575-584. https://revistas.javeriana.edu.co/index.php/ revPsycho/article/view/3810

García, F., Jaramillo, C., Martínez, A., Valenzuela, I., \& Cova, F. (2014). Respuestas psicológicas ante un desastre natural: estrés y crecimiento postraumático. Liberabit, 20(1), 121130. http://ojs3.revistaliberabit.com/publicaciones/revistas/ RLE_20_1_respuestas-psicologicas-ante-un-desastre-natu ral-estres-y-crecimiento-postraumatico.pdf

Gaudreau, P. (2018). Active Coping Strategies. En V. ZeiglerHill \& T. Shackelford (Eds), Encyclopedia of Personality and Individual Differences (pp. 34-36). Springer. https:// doi.org/10.1007/978-3-319-28099-8_1119-1

Grothmann, T., \& Reusswig, F. (2006). People at risk of flooding: why some residents take precautionary action while others do not. Natural hazards, 38(1-2), 101-120. https:// doi.org/10.1007/s11069-005-8604-6

Gruev-Vintila, A., \& Rouquette, M.-L. (2007). Social thinking about collective risk: How do risk-related practice and personal involvement impact its social representations? Journal of Risk Research, 10(4), 555-581. https://doi. org/10.1080/13669870701338064

Guillard, M., Navarro, O., \& Fleury-Bahi, G. (2019). Flooding experience and assessment of climate change: implication of psychological distance, risk perception and place attachment. Psyecology, 10(3), 287-312. https://doi.org/10.1080/ 21711976.2019.1622347

Henríquez, C, Aspee, N., \& Quense, J. (2016). Zonas de catástrofe por eventos hidrometeorológicos en Chile y aportes para un índice de riesgo climático. Revista de geografía Norte Grande, (63), 27-44. https://dx.doi.org/10.4067/ S0718-34022016000100003

Ho, M., Shaw, D., Lin, S., \& Chiu, Y. (2008). How do disaster characteristics influence risk perception? Risk Analysis: An International Journal, 28(3), 635-643. https://doi. org/10.1111/j.1539-6924.2008.01040.x

Hobfoll, S. E. (1998). Stress, culture, and community: The psychology and philosophy of stress. Plenum Press.

Intergovernmental Panel on Climate Change [IPCC]. (2014). Climate change 2014: Impacts, adaptation, and vulnerability. ipcc's fifth anual report'. Intergovernmental Panel on Climate Change. https://www.ipcc.ch/report/ar5/wg2/

Kellens, W., Terpstra, T., \& de Maeyer, P. (2013). Perception and communication of flood risks: a systematic review of empirical research. Risk Analysis: An International Journal, 33(1), 24-49. https://doi.org/10.1111/j.1539-6924.2012.01844.x

Kellens, W., Zaalberg, R., Neutens, T., Vanneuville, W., \& De Maeyer, P. (2011). An analysis of the public perception of flood risk on the Belgian coast. Risk analysis: an official publication of the Society for Risk Analysis, 31(7), 1055-1068. https://doi.org/10.1111/j.1539-6924.2010.01571.x

Lechowska, E. (2018). What determines flood risk perception? A review of factors of flood risk perception and relations between its basic elements. Natural Hazards, 94(3), 13411366. https://doi.org/10.1007/s11069-018-3480-z

León, O., \& Montero, I. (2015). Métodos de investigación en psicología y educación: Las tradiciones cuantitativa y cualitativa (4. ${ }^{\mathrm{a}}$ ed.). McGraw-Hill.

Lindell, M., \& Perry, R. (2012). The protective action decision model: theoretical modifications and additional evidence. Risk Analysis: An International Journal, 32(4), 616-632. https://doi.org/10.1111/j.1539-6924.2011.01647.x

Little, B. (2018). Passive Coping Strategies. En V. Zeigler-Hill y T. Shackelford T. (Eds), Encyclopedia of Personality and Individual Differences. Springer. https://doi. org/10.1007/978-3-319-28099-8_1867-1

López-Vázquez, E., \& Marván, M. L. (2004). Validación de una escala de afrontamiento frente a riesgos extremos. Salud Pública de México, 46(3), 216-221. https://www.redalyc. org/pdf/106/10646305.pdf

Ministerio de Desarrollo Social y Familia de Chile. (2010). Cuestionario encuesta post-terremoto. 2010. http://www. desarrollosocialyfamilia.gob.cl/pdf/informe-encuesta-postterremoto.pdf

Moos, R., \& Billings, A. (1982). Conceptualizing and measuring coping resource and processes. En L. Goldberger y S. Breznitz (Eds.), Handbook of stress: Theoretical and clinical aspects (pp. 212-230). Free Press.

Muñoz-Duque, L., \& Arroyave, O. (2017). Percepción del riesgo y apego al lugar en población expuesta a inundación: un estudio comparativo. Pensamiento Psicológico, 15(2), 79-92. https://doi.org/10.11144/Javerianacali.PPSI15-2.pra 
Navarro, O., Chaves-Castaño, L., Noreña Betancur, M., \& Piñeres-Sus, J. (2016). Percepción del riesgo y estrategias de afrontamiento en población expuesta y no expuesta al riesgo de inundación. Interamerican Journal of Psychology, 50(3), 331-346. https://doi.org/10.30849/rip/ijp.v50i3.62

Navarro, O., Restrepo-Ochoa, D., Muñoz-Duque, L.A., Zapa-Perez, K., Ameline, A., Mercier, D., \& Fleury-Bahi, G. (2020). Determinants of coping strategies in two types of natural hazards: flash floods and coastal flooding. International Journal of Disaster Risk Reduction, 46, 101514, https://doi.org/10.1016/j.ijdrr.2020.101514

Newsom, J. T. (2018). Minimum sample size recommendations (Psy 523/623 structural equation modeling, Spring 2018). Manuscript. http://web.pdx.edu/ newsomj/semclass/ho_es timate.pdf

Olivos-Jara, P., Navarro-Carrascal, O., \& Loureiro, A. (2020). Cómo afrontar una catástrofe: Percepción de riesgo y factores psicosociales de la adaptación. Ediciones de la Universidad de Castilla-La Mancha.

Pardo, A., Ruiz, M. A., \& San Martín, R. (2014). Análisis de datos en ciencias sociales y de la salud. Síntesis.

Paton, D., Smith, L., Daly, M., \& Johnston, D. (2008). Risk perception and volcanic hazard mitigation: Individua and social perspectives. Journal of Volcanology and Geothermal Research, 172, 179-188. https://doi.org/10.1016/j. jvolgeores.2007.12.026

Preacher, K., \& Hayes, A. (2008). Asymptotic and resampling strategies for assessing and comparing indirect effects in multiple mediator models. Behavior research methods, 40(3), 879-891. https://doi.org/10.3758/BRM.40.3.879

Raaijmakers, R., Krywkow, J., \& van der Veen, A. (2008). Flood risk perceptions and spatial multi-criteria analysis: an exploratory research for hazard mitigation. Natural hazards, 46(3), 307-322. https://doi.org/10.1007/s11069-007-9189-z

Ruiter, R., Kessels, L., Peters, G., \& Kok, G. (2014). Sixty years of fear appeal research: Current state of the evidence. International Journal of Psychology, 49(2), 63-70. https:// doi.org/10.1002/ijop.12042

Sandoval-Díaz, J, \& Cuadra-Martínez, D. (2020). Vulnerabilidad social, severidad subjetiva y crecimiento postraumático en grupos afectados por un desastre climatológico. Revista de Psicología (Santiago), 29(1), 42-56. https://dx.doi. org/10.5354/0719-0581.2020.58002

Sandoval-Díaz, J. (2020). Vulnerabilidad-resiliencia ante el proceso de riesgo-desastre: Un análisis desde la ecología política. Polis (Santiago), 19(56), 138-154. https://dx.doi. org/10.32735/s0718-6568/2020-n56-1527

Sandoval-Díaz, J., Karmelic-Pavlov, V., Tello-Cabrera, S., Chaparro-Guzmán, M., Gaete-Bastías, G., \& AlfaroSaldívar, K. (2020). Subjetividad y medios de vida sostenibles de hogares vulnerados por un desastre hidrometereológico en la región de Atacama de Chile. Ciencias Psicológicas, 14(2), e2287. https://dx.doi.org/10.22235/ cp.v14i2.2287
Sandoval-Díaz, J., Rojas-Páez, L., Villalobos-Soublet, M., Sandoval-Díaz, C., Moraga, F., \& Aguirre, N. (2018). De organización vecinal hacia la gestión local del riesgo: diagnóstico de vulnerabilidad y capacidad. Revista INVI, 33(92), 155-180. https://dx.doi.org/10.4067/ S0718-83582018000100155

Sapiains, R., \& Ugarte, A. (2017). Contribuciones de la Psicología al abordaje de la dimensión humana del cambio climático en Chile (Primera parte). Interdisciplinaria, 34(1), 91-105. http://www.ciipme-conicet.gov.ar/ojs/in dex.php?journal $=$ interdisciplinaria\&page $=$ article $\& o p=v i e$ w\&path $\% 5 \mathrm{~B} \% 5 \mathrm{D}=280 \&$ path $\% 5 \mathrm{~B} \% 5 \mathrm{D}=36$

Servicio Nacional de Geología y Minería de Chile [SERNAGEOMIN]. (2015). Zonas afectadas por inundación y flujos de detritos (aluvión). zonas propuestas para: evacuación, campamentos y acopio en Copiapó y Paipote. [Mapa]. http://sitiohistorico.sernageomin.cl/pdf/mapageo/AluvionPaipote-comuna-Copiapo-region-Atacamamarzo2015.pdf

Siclari, P. (2020). Amenazas de cambio climático, métricas de mitigación y adaptación en ciudades de América Latina y el Caribe. Documentos de Proyectos (LC/TS.2020/185). Comisión Económica para América Latina y el Caribe (CEPAL). https://repositorio.cepal.org/handle/11362/46575

Siegrist, M., \& Gutscher, H. (2006). Flooding risks: A comparison of lay people's perceptions and expert's assessments in Switzerland. Risk Analysis, 26(4), 971-979. https://doi. org/10.1111/j.1539-6924.2006.00792.x

Slovic, P. (2000). The perception of risk. Earthscan.

Sobel, M. (1982). Asymptotic Confidence Intervals for Indirect Effects in Structural Equation Models. Sociological Methodology, 13, 290-312. http://dx.doi. org $/ 10.2307 / 270723$

Tannenbaum, M., Hepler, J., Zimmerman, R., Saul, L., Jacobs, S., Wilson, K., \& Albarracín, D. (2015). Appealing to fear: A meta-analysis of fear appeal effectiveness and theories. Psychological Bulletin, 141(6), 1178-1204. http://dx.doi. org/10.1037/a0039729

Terpstra, T., Gutteling, J., Geldof, G., \& Kappe, B. (2005). The perception of flood risk and water nuisance. 45th European Congress of the Regional Science Association. Vrije Universiteit Amsterdam. Amsterdam.

Tompkins, M., Bjälkebring, P., \& Peters, E. (2018). Emotional Aspects of Risk Perceptions. En M. Raue, E. Lermer y B. Streicher (Eds.), Psychological Perspectives on Risk and Risk Analysis: Theory, Models, and Applications (pp. 109130). Springer.

UN Office for Disaster Risk Reduction [UNDRR]. (2015). Marco de Sendai para la Reducción del Riesgo de Desastres 20152030. UNDRR.

UN Office for Disaster Risk Reduction [UNDRR]. (23 de enero de 2019). 2018: 60 millones de personas resultaron afectadas por diversos eventos meteorológicos extremos. https://eird.org/americas/ 
noticias/2018-60-millones-de-personas-resultaron-afecta das-por-diversos-eventos-meteorologicos extremos.html\#. XatY65NKg_V

Wachinger, G., Renn, O., Begg, C., \& Kuhlicke, C. (2013). The risk perception paradox-implications for governance and communication of natural hazards. Risk Analysis, 33(6), 1049-1065. https://doi.org/10.1111/j.1539-6924.2012.0194 2.x

Wlodarczyk, A., Basabe, N., Páez, D., Reyes, C., Villagrán, L., Madariaga, Palacios, C., \& Martínez, F. (2016). Communal Coping and Posttraumatic Growth in a Context of Natural Disasters in Spain, Chile, and Colombia.
Cross-Cultural Research, 50(4), 325-355. https://doi. org/10.1177/1069397116663857

Zapa-Pérez, K., Navarro, O., \& Rendón-Rivera, A. (2017). Modelo de análisis de la vulnerabilidad psicosocial en la gestión del riesgo de desastres. Revista de Gestão Social e Ambiental, 11(2), 91-110. https://doi.org/10.24857/rgsa. v11i2.1309 